\title{
Asymmetric aldol reactions catalyzed by the promiscuous aldo-ketoreductase enzyme
}

\begin{abstract}
The promiscuous aldo-ketoreductase (AKR) enzyme is used as a sustainable biocatalyst for the first time to catalyze asymmetric aldol reactions in aqueous medium. The reactions between aromatic aldehydes and cyclic/acyclic ketones give the corresponding products in moderate yields and enantioselectivities in the presence of water. The influence of solvents, the mole ratio of substrates, and enzyme concentration are investigated. The mechanism of the AKR1A1-catalyzed aldol reaction is also discussed.
\end{abstract}

Keyword: Promiscuous enzymes; Aldo-ketoreductase; Asymmetric; Aldol reaction 